\title{
EL ORO Y SUS ALEACIONES EN LA ORFEBRERÍA PRERROMANA
}

\author{
POR \\ IGNACIO MONTERO Y SALVADOR ROVIRA
}

\begin{abstract}
RESUMEN
El estudio sistemático de los análisis de objetos de oro de la P. Ibérica permite observar tendencias y agrupamientos que personalizan los distintos períodos culturales desde el Bronce Antiguo hasta el Hierro II. Mientras el Bronce Antiguo y Medio se caracteriza por el empleo de oro nativo, en el Final fue práctica cotidiana la aleación oro-plata. El Período Orientalizante y las culturas del Hierro diversifican las tradiciones de orfebre bajo los influjos tecnológicos del Mediterráneo oriental, marcando una clara diferencia entre la cultura Ibérica y la del Hierro del Noroeste.
\end{abstract}

\section{SUMMARY}

The systematic study of metal analysis of gold objects from de Iberia Peninsula allows us to define some tendences and clusters that confer personality to cultural periods from Early Bronze to Late Iron Age. Whilst Early and Middle Bronce emphasize the use of native gold, in Late Bronze Age the gold/silver alloying practice was common. The Orientalizing Period and Iron cultures diversify gold-smith traditions under technological influences arrived from the Eastern Mediterranean, drawing a sharp contrast between the Iberic culture and the iron one in the northwest of Spain.

\section{INTRODUCCIÓN}

La orfebrería de todos los tiempos ha sido siempre objeto predilecto de investigación, actuando como polos de atracción la propia calidad intrínseca de las pequeñas y grandes obras de arte confeccionadas y el elevado valor simbólico-real de la materia empleada: el metal precioso. Esto último sigue suponiendo una de las mayores trabas a la hora de plantear investigaciones de índole tecnológica (siempre supuestamente agresivas para los objetos), generan- 
do prejuicios a veces irreductibles en propietarios y conservadores de museos. Con todo, en los últimos cuarenta años se ha ido reuniendo un conjunto de datos sobre la calidad del oro de las producciones de orfebre halladas en la Península, suficiente para encarar con ciertas garantías de éxito una ordenación sistemática de las aleaciones y su evolución a lo largo de la Prehistoria reciente.

Utilizaremos dos fuentes principales: 446 análisis publicados por Hartmann ' y 24 realizados por nosotros dentro del programa Arqueometalurgia de la Península Ibérica.

\section{EL ORO Y SUS ALEACIONES}

Como es sabido, el oro puro es un metal de color amarillo, brillante cuando tiene la superficie pulida e inalterable ante los agentes corrosivos habituales. Ocupa el lugar más elevado dentro de la serie galvánica $\left(\mathrm{E}_{\mathrm{o}}=-1,5 \mathrm{~V}\right.$ a $\left.25^{\circ} \mathrm{C}\right)$, siendo, por tanto, el metal más noble. Sus cualidades mecánicas más características son blandura, ductilidad y maleabilidad. El punto de fusión se halla en $1063^{\circ} \mathrm{C}$, temperatura relativamente elevada que se rebaja de manera sensible en algunas aleaciones.

La plata ligada al oro tiene la virtud de aclarar el color dorado, pasando sucesivamente a tonalidades cada vez más alimonadas cuando la tasa de plata remonta el $20 \%$ (electrum), hasta el blanco amarillento de las aleaciones de oro bajo con plata. Estos dos metales ligan perfectamente (la plata funde a $960,5^{\circ} \mathrm{C}$ ), pero sus aleaciones mantienen intervalos de solidificación relativamente altos. Así, una aleación $80 / 20$ no comienza a fundir hasta los $1.040{ }^{\circ} \mathrm{C}$, tan sólo unos $20^{\circ}$ menos que el oro puro.

El cobre también liga perfectamente con el oro. Tiene el efecto de cobrizar el oro de la aleación, virando el oro hacia tonos más cálidos. Pero sin duda uno de los efectos de mayor repercusión tecnológica es el notable descenso de la temperatura de fusión: una aleación 80/20 funde rápidamente a $890^{\circ} \mathrm{C}$, a pesar de que el cobre solo lo hace a $1.083^{\circ} \mathrm{C}$.

Determinadas aleaciones hacen más trabajable el oro, soportando mejor los esfuerzos de laminación, repujado, etc. Por tanto, el uso y control de las aleaciones pueden ser, en principio, indicadores del dominio y desarrollo tecnológico, facilitando el trabajo al artesano, dando más calidad a sus manufacturas y repercutiendo de alguna manera en el sistema económico. Todas estas razones obligan a elaborar un capítulo sobre aleaciones, dentro de la historia de la metalurgia. Ahora bien, para poder hablar sobre control y uso de aleaciones deberemos, en primer lugar, acordar qué constituye una aleación intencionada, qué elementos forman parte de la aleación ysi es técnicamente viable su preparación. Ello nos lleva inevitablemente a tratar la cuestión de la composición original de la materia prima.

' Salvo mención específica, la mayoría de los datos analíticos proceden de A. HaRTMAnN, Prähistorische Goldfunde aus Europa II. Spektralanalytische Unterschungen und deren Auswertunq, S.A.M. 5, Gebr. Mann, Verlag, Berlín, 1982. A sus tablas remitimos en adelante, aliviando así el texto de citas repetitivas. 


\section{COMPOSICIÓN DEL ORO NATIVO}

El oro se diferencia del resto de los metales usados en la metalurgia antigua por ser aprovechado en estado nativo. Esta circunstancia y su recolección mediante una sencilla técnica de lavado de las arenas auríferas con bateas, hacen innecesarios grandes conocimientos técnicos previos para conseguirlo, cuando existe en el medio natural. Por el conrario, la explotación minera del oro supone una gran inversión de esfuerzo, técnica y medios, y no parece comenzar hasta época romana. De ello se sigue que la gran mayoría de los objetos prerromanos de oro proceden casi con seguridad del oro aluvial ${ }^{2}$.

La composición del oro aluvial puede ser muy variable, especialmente por su contenido en plata, metal que junto con el oro va a ser elemento clave de las aleaciones. Por desgracia la información disponible sobre la composición del oro nativo peninsular es muy pobre — por no decir que nula-. Están por hacer los estudios analíticos con muestras de las distintas cuencas auríferas y tan sólo contamos para el oro antiguo con datos genéricos extraídos de los comentarios de Plinio ${ }^{3}$, quien aseguraba "que el oro español tiene siempre plata en proporción variable, a veces hasta un décimo o un octavo". Según la cita, el oro debería presentar del 10 al $12,5 \%$ de plata. Sin embargo el único análisis que conocemos, procedente de Guadamar (Alicante) ${ }^{4}$, da oro bastante puro, y existen materiales del Calcolítico y Bronce Antiguo como la placa del dolmen de Matarrubilla (Sevilla), la lámina de la Loma de Belmonte (Almería) y uno de los torques de Caldas de Reyes (Pontevedra), con porcentajes entre el 3 y el $6 \% \mathrm{Ag}$. Con todo, y a pesar de la variabilidad observada en los objetos analizados, ciertamente hay un gran número de piezas de la Edad del Bronce que se sitúan en torno al intervalo atribuido por Plinio al oro peninsular.

Por lo que se conoce del oro en otras regiones del mundo 5 , la tasa de plata puede oscilar desde unas pocas décimas hasta casi el $40 \%$ en algunas ocasiones y países. Estas asociaciones naturales de oro con más del $20 \%$ de plata son las denominadas por los clásicos electrum o electro. La elevada cantidad de plata que es posible encontrar en el oro nativo hace difícil distinguir buen número de aleaciones intencionadas $\mathrm{o}$, al contrario, podemos atribuir a la voluntad del orfebre lo que es en realidad un producto natural.

En España muy pocos objetos de los primeros períodos metalúrgicos presentan valores superiores al 20-25\% Ag, y de ellos, las dos muestras analizadas por Siret ${ }^{6}$ son de época argárica y se pueden considerar aleaciones artificiales con plata, metal también utilizado en aleación con cobre en dicha época ${ }^{7}$, más bien que electro natural. El mismo Siret comenta que tales objetos son los de color más claros, dando a entender que el resto de las piezas de oro

${ }^{2}$ F. J. SÁnchez-PAlencia y L. C. PÉrez, «Los yacimientos auríferos de la Península Ibérica. Posibilidades de explotación en la Antigüedad", en El oro en la España Prerromana, Revista de Arqueología, 1989, pp. 16-23.

${ }^{3}$ PLINIO EL VIEJO, Naturae historiarum libri, XXXIII, 23-80.

4 J. N. BARRANDÓN, «L'Or: du minerai au métal. L'Apport des analyses», en Minería y metalurgia en las antiguas civilizaciones mediterráneas y europeas, vol. II, Ministerio de Cultura, Madrid, 1989, p. 59.

${ }^{5}$ R. F. TYLECOTE, The early history of metallurgy in Europa. Longman, London, 1987, tabla 35; Barrandon, op. cit., n. 4, p. 53.

${ }^{6}$ E. y L. SiRET, Las primeras edades del metal en el Sudeste de España, Barcelona, 1890, p. 296.

7. I. Montero, Estudio arqueometalúrgico en el Sudeste de la Península Ibérica. Tesis Doctoral, Facultad e G." e Historia, Univ. Complutense, leída en 1991, inédita, p. 481. 
de los yacimientos del sureste que excavó tendrían menor cantidad de plata ${ }^{8}$. Otros argumentos en favor de la baja tasa de plata del oro nativo de esta región pueden extraerse de los tres análisis practicados por nosotros a una lámina y dos cuentas cilíndricas de la Loma de Belmonte, dando entre 2,9 y $6,4 \% \mathrm{Ag}$. El tercer caso de oro con más del $25 \%$ de plata es un brazalete de Lamela de Silleda que, por llevar también un $4,1 \%$ de cobre, ofrece pocas dudas de ser una aleación artificial.

Además de plata, el oro nativo suele llevar pequeñas cantidades de cobre. Salvo excepciones detectadas por ahora en Alemania, norte de Italia y Checoslovaquia, en donde el cobre alcanza el $3,5 \%$, los valores normales no superan el $1 \%$. Tal parece ser el caso de la Península pues los materiales antiguos, tanto en la serie de Hartmann como en la nuestra, se mantienen por debajo de dicha cifra.

Podemos, pues, de manera tentativa, fijar como límites convencionales por encima de los cuales podemos hablar de aleaciones artificiales las cifras del $25 \% \mathrm{Ag}$ y $1 \% \mathrm{Cu}$, límites que deberían ser corroborados mediante análisis de oro nativo peninsular. Tales aleaciones pueden lograrse ligando oro con plata, con cobre o con dos elementos de manera conjunta por medio de plata ligada previamente con cobre, proceso este último que no resultaría anómalo, dado que era una aleación conocida desde época argárica ${ }^{10}$.

Junto a la plata y el cobre pueden acompañar al oro otras impurezas, entre las que destacaremos el estaño y el platino por las interpretaciones a que han dado lugar. La presencia de estaño suele ser frecuente en el oro aluvial; pero ello no quiere decir que todo el oro con algo de estaño es de origen aluvial, mientras que el que no lo lleva procede de una veta encajada. El estaño puede ir asociado al oro de veta, en cuyo caso las pepitas arrastradas por la erosión contendrán dicho elemento. Si la veta no lo contiene pero sí las arenas fluviales por proceder de algún metalotecto estannífero, las partículas de oro presentes disolverán parte del estaño quedando contaminadas. Finalmente, si la veta original y las arenas no son estanníferas, tampoco lo será el oro recuperado en sus placeres.

En cuanto al platino, presente en algunos depósitos y ausente en otros, no resulta conveniente emplearlo como criterio discriminante para establecer orígenes y posibles relaciones comerciales por la carencia antes aludida de estudios analíticos de la calidad del oro en las distintas zonas auríferas de la Península. Mientras tanto, conviene tomar con cierta reserva la sugerencia lanzada por Hartmann y Kalb " sobre importaciones de materia prima del Mediterráneo oriental (donde el platino es impureza frecuente de la orfebrería), en época ibérica, por el hecho de haber detectado platino en algunas piezas levantinas. El análisis del oro alicantino de Guadamar, antes comentado, titula el elemento en cuestión, por lo que nada tiene de extraña su presencia en la orfebrería ibérica. Las importaciones, si las hubo, deberán argumentarse desde otras perspectivas y no sólo basadas en la vía analítica.

En resumen, a través de las impurezas del oro no resulta prudente por el momento determinar zonas de aprovechamiento y relaciones con la materia prima, al tiempo que tampoco

${ }^{8}$ SIREt, op. cit, n. 6, p. 296.

${ }^{9}$ TYLECOTE, op. cit., n. 5; Barrandon, op. cit., n. 4, p. 59.

${ }^{10} \mathrm{Vid} . \mathrm{n} .7$.

"A. HARTMANN y P. KALB, «Investigaciones espectroanalíticas sobre hallazgos hispánicos de oro", Arch. Prehist. Levantina, vol. XII, 1969, p. 95. 
es útil la clasificación en grupos efectuada por Hartmann 12, basada exclusivamente en intervalos de composición de las tasas de impurezas. Unicamente cuando el estudio de los análisis se elabora en función de la cronología y de la localización geográfica de los objetos, observando las tendencias, pueden extraerse algunas conclusiones de interés y apreciar el cambio y la evolución tecnológica seguida. El estudio individualizado de la composición por piezas puede venir afectado por muchas circunstancias, entre otras por la frecuente reutilización del oro.

\section{EVOLUCIÓN TECNOLÓGICA}

Como ya se ha indicado, la fuente más numerosa de datos analíticos para este trabajo se ha tomado de los análisis publicados por Hartmann en 1982. De ellos hemos seleccionado aquellos en los que se ha podido establecer la procedencia concreta y una cronología precisa. No se han tomado en cuenta por el momento los materiales portugueses ni otros procedentes de yacimientos españoles que no hemos identificado satisfactoriamente. También se han desechado aquellos análisis de Hartmann en los que la determinación de la plata resulta imprecisa, cuando tal imprecisión imposibilitaba asignarles alguno de los intervalos de clase empleados en nuestra clasificación.

Los datos disponibles manifiestan que, desde un momento relativamente temprano, se están empleando aleaciones intencionadas de oro, aunque con una frecuencia pequeña, tanto en el norte como en el sur de la Península: un hilo de El Argar (Antas, Almería) contiene algo más del $34 \% \mathrm{Ag}$; un anillo espiral del Cerro de la Virgen (Orce, Granada), 2,1 \% Cu; uno de los brazaletes de Lamela de Silleda (Pontevedra), 4,1 \% Cu. Por tanto, en algún momento aún no bien acotado del Bronce Medio los orfebres indígenas comenzaron a ensayar la manera de bajar la ley del oro.

El primer cambio importante en la composición del oro llega con el Bronce Final. Según los histogramas de frecuencia relativas de las figuras 1 y $2{ }^{1.3}$ existe una clara diferencia de los contenidos de cobre y plata con respecto a épocas anteriores. Mientras en el Bronce Antiguo y Medio las impurezas de cobre se sitúan mayoritariamente en los intevalos bajos $(<0,1 \%$ y 0,1-0,25\%), en el Bronce Final se concentran a partir del intervalo 0,26-0,5\%. Además, la frecuencia de objetos con más del $1 \% \mathrm{Cu}-\mathrm{y}$ por tanto presuntamente de oro aleado- se incrementa notablemente, alcanzando el $25 \%$ de los materiales en el Bronce Final frente al $5 \%$ de etapas precedentes. Con posterioridad al Bronce Final, el $80 \%$ de los análisis tienen más del $1 \%$ de cobre.

En relación con la tasa de plata, la figura 2 muestra un desplazamiento hacia la derecha de los objetos del Bronce Final. Ahora los intervalos más frecuentados son el 10-15\% y 15 $20 \% \mathrm{Ag}$, mientras durante el Bronce Antiguo y Medio lo era el intervalo 5-10\%. Las causas de estos cambios en los hábitos compositivos no son fáciles de explicar. Podría pensarse,

\footnotetext{
${ }^{12}$ HARTMANN, op. cit., n. 1.

${ }^{13}$ En el contenido de cobre no están incluidas las piezas analizadas dentro del Programa de Arqueometalurgia debido a que el límite de detección del espectrómetro utilizado no es tan preciso para este elemento, detectándolo como trazas no cuantificables cuando la cantidad es inferior a $0,25 \% \mathrm{Cu}$.
} 
en principio, y dada la mayor abundancia de objetos de oro, que la respuesta del orfebre ante la demanda creciente fue aumentar el rendimiento del metal bajando la ley. Pero, aunque la economía de mercado funcionara según pautas paralelizables con las del libre mercado - cosa poco probable tratándose de un bien escaso, atesorable y elitista—, nos faltaría para poder apuntalar la opinión un conocimiento más detallado de la circulación de metal y de la optimización de la explotación de los recursos disponibles. Por el contrario, existen textos contemporáneos y aun anteriores, de otras latitudes, que hablan expresamente de la estricta vigilancia y fiscalización a que son sometidos los artesanos del metal para evitar fraudes ${ }^{14}$. Por otro lado, la figura 1 indica que aún en el Bronce Final hay poca tendencia a ligar con cobre pero, al mismo tiempo, las impurezas de cobre aumentan cuantitativamente. Ello es un reflejo, junto con el aumento de la plata, de la frecuente refundición de joyas amortizadas. Efectivamente, la reutilización de alguna pieza más cobrizada junto con otras porciones de oro más limpio hará subir la tasa media de cobre. Hay testimonios arqueoló-
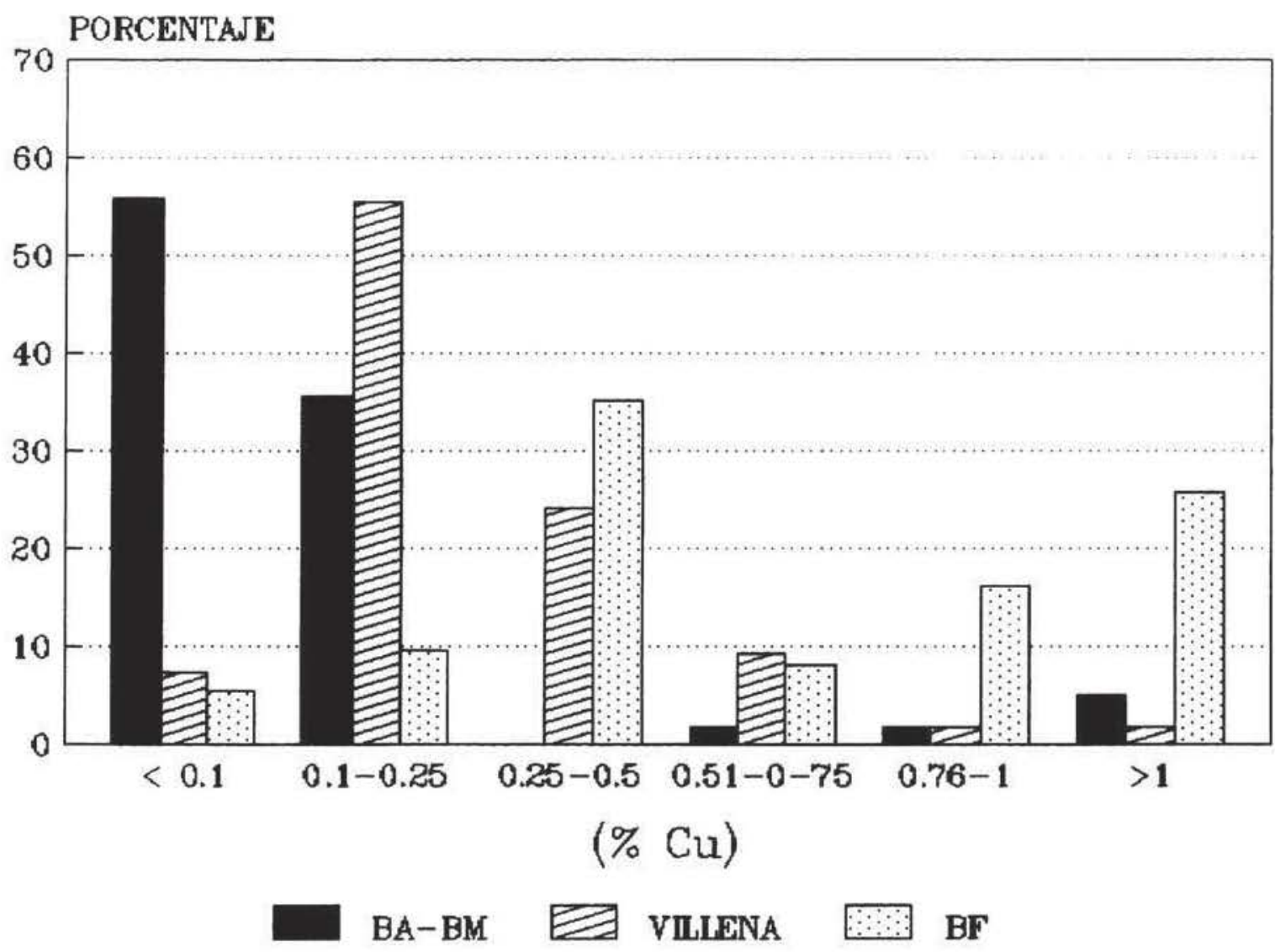

Figura 1. Contenido en cobre (Frecuencias relativas).

${ }^{14}$ Datos a este respecto se encuentran en las tablillas micénicas de Pilos, citadas por J. CHADWICK, El mundo micénico, Alianza, Madrid, 1977, pp. 185-186. 
gicos de la existencia de estos tesorillos de fundidor, como son los casos de Bodonal de la Sierra y Bélmez ${ }^{15}$.

El incremento de la tasa media de plata probablemente se deba a razones tecnológicas: la búsqueda y ensayo de mejores aleaciones para trabajar más cómodamente el oro. Recuérdesde que el oro industrial de 22 quilates contiene $91,6 \%$ Au y el de 18 quilates (el más empleado por reunir las mejores cualidades) $75 \%$ Au. Otros avances tecnológicos de la orfebrería del Bronce Final fueron el empleo de moldes para vaciado (varillas, anillos, brazaletes, torques) y de una incipiente técnica de soldadura ${ }^{16}$.

Entre los materiales del Bronce Final cabe estudiar aparte el tesoro de Villena, conjunto numeroso y representativo en sí mismo. Si observamos la distribución del contenido de im-

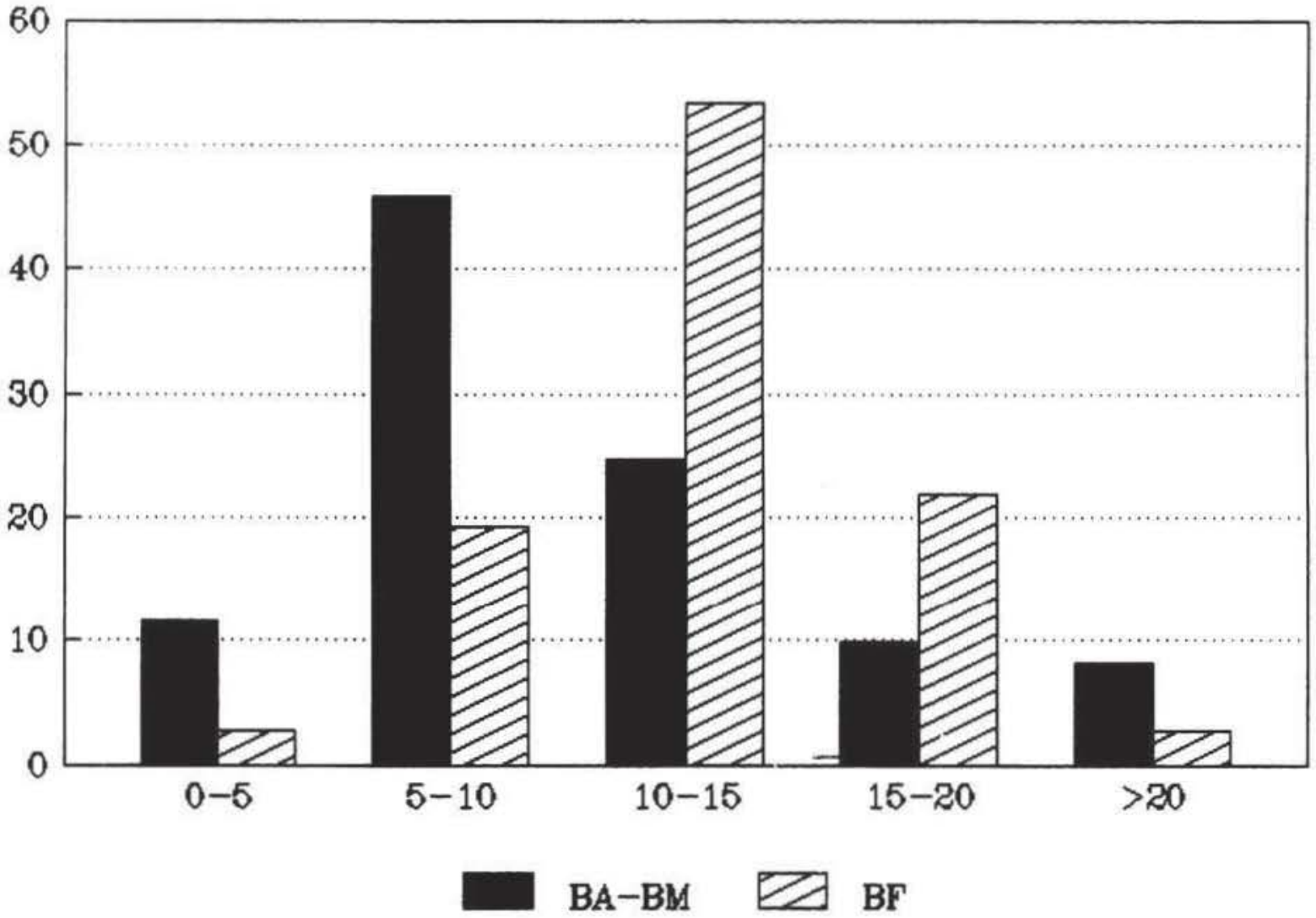

Figura 2. Contenido en plata (Frecuencias relativas).

${ }^{15}$ M. L. RUIZ-VÁLVEZ, «La orfebrería del Bronce Final. El poder y su ostentación», en El oro en la España prerromana, Revista de Arqueología, 1989, p. 46-57.

${ }^{16}$ A. PEREA, «Tecnología y métodos de estudio», en El oro en la España prerromana, Revista de Arqueología, 1989, p. 27. 
purezas de cobre en la figura 1 obtendremos una situación intermedia en relación con los materiales del Bronce Antiguo y Medio y el resto de los del Bronce Final. El contenido medio de plata, $8,6 \%$, contrasta también con el $12,78 \%$ del Bronce Final, quedando más próximo pero por encima del contenido de los materiales del Bronce Antiguo y Medio. Esta diferencia se hace más llamativa si comparamos este conjunto con el del Cabezo Redondo, también en término de Villena, que presenta mayor contenido medio de cobre y plata, en cifras muy similares a las medias del Bronce Final. Esta situación intermedia de la calidad del oro del tesoro de Villena permite terciar en la polémica acerca de la cronología de este singular hallazgo. ¿Nos hallamos ante unos materiales importados o, al menos, fabricados con oro de distinta procedencia que la del resto de materiales promediados? ¿Se trata de un atesoramiento con mayor antigüedad que la hasta ahora atribuida condicionada por la presencia de hierro, nuevo metal que pudo haber sido incorporado al conjunto en un momento tardío en relación con el de fabricación de las piezas de oro? ¿O, simplemente, se empleó mayor cantidad de oro nativo que de recuperado, al contrario de lo que parece ser hábito común de otros conjuntos del Bronce Final?

La orfebrería del Período Orientalizante, donde ya las composiciones con cobre son mayoritarias, ofrece otro notable contraste siendo los rasgos principales que la caracterizan su diversidad y variedad. Mientras que hasta este momento veíamos una relativa homogeneidad en la composición de las distintas zonas que nos permitía sistematizar de forma general los períodos, ahora resultan homogéneos los conjuntos individualizados pero diferentes unos de otros, como se observa en los seleccionados para el cuadro 1. Ello desaconseja el tratamiento conjunto de los datos, ya que de hacerlo obtendríamos unos resultados dependientes del número de piezas analizadas de cada conjunto. El que los candelabros de Lebrija tengan plata pero no cobre, que Trayamar tenga algo más de plata y también cobre, que el tesoro de El Carambolo presente menor cantidad de plata que los anteriores, pero cobre similar a Trayamar, o que Jávea no tenga apenas plata pero sí bastante cobre, mientras que en La Aliseda se combinan varios modelos, simplemente está confirmando la diversidad de influencias y tradiciones que entran en juego y se entrecruzan durante el Período Orientalizante. A ellas debemos la introducción en la Península de las técnicas de filigrana y granulado que conferirán a las labores una belleza y una complejidad en los diseños desconocida hasta entonces ${ }^{17}$.

Tras este período de diversidad y variación vuelve a establecerse una norma predominante en las producciones de orfebre de la segunda mitad del primer milenio a. C., norma que será distinta para las dos zonas peninsulares: el noroeste y el mundo ibérico o mediterráneo. Por lo que respecta a esta última, la composición en plata es similar en toda la geografía de los pueblos ibéricos, como refleja la figura 3 donde se comparan los materiales ibéricos con los del taller de Cádiz. Esta similitud en los tipos de aleación, que implica unidad en este rasgo tecnológico, en nada se opone a las evidentes diferencias de estilo y modo de trabajar el metal dentro complejo mundo ibérico, de las que sin duda es ejemplo clarísico la orfebrería gaditana.

${ }^{17}$ Estos y otros aspectos de la tecnología han sido tratados en profundidad por A. PEREA, op. cit., n. 17 , p. 29-3r. 
OBJETOS DE ORO ORIENTALIZANTES

\begin{tabular}{|c|c|c|c|}
\hline YACIMIENTO & $\% \mathrm{Ag}$ & $\% \mathrm{Cu}$ & OBJETO \\
\hline El Acebuchal & $30-35$ & 4,8 & Aro abierto \\
\hline El Acebuchal & $30-35$ & 6,0 & Aro abierto \\
\hline El Acebuchal & $30-35$ & 6,5 & Anillo \\
\hline El Acebuchal & 16 & 1,6 & Remache \\
\hline YACIMIENTO & $\% \mathrm{Ag}$ & $\% \mathrm{Cu}$ & OBJETO \\
\hline El Carambolo & 10 & 3,4 & Lámina \\
\hline El Carambolo & 5 & 3,6 & Placa \\
\hline El Carambolo & 10 & 4,5 & Lámina \\
\hline El Carambolo & $3-5$ & 1,8 & Pectoral \\
\hline YACIMIENTO & $\% \mathrm{Ag}$ & $\% \mathrm{Cu}$ & OBJETO \\
\hline Jávea & 1 & 5,3 & Diadema \\
\hline Jávea & 9 & 5,9 & Cadenilla \\
\hline Jávea & 1 & 4,8 & Cadenilla \\
\hline Jávea & 1 & 5,6 & Cadena \\
\hline Jávea & 1 & 4,6 & Anillo \\
\hline Jávea & 1 & 3,6 & Colgante con cadena \\
\hline YACIMIENTO & $\% \mathrm{Ag}$ & $\% \mathrm{Cu}$ & OBJETO \\
\hline Lebrija & 15 & 0,48 & Candelabro \\
\hline Lebrija & 11 & 0,52 & Candelabro \\
\hline Lebrija & 18 & 0,45 & Candelabro \\
\hline Lebrija & 15 & 0,40 & Candelabro \\
\hline Lebrija & 11 & 0,42 & Candelabro \\
\hline Lebrija & 15 & 0,42 & Candelabro \\
\hline YACIMIENTO & $\% \mathrm{Ag}$ & $\% \mathrm{Cu}$ & OBJETO \\
\hline Trayamar & $20-25$ & 2,3 & Medallón \\
\hline Trayamar & 40 & 11,5 & Anillo \\
\hline Trayamar & $20-25$ & 3,4 & Pendiente \\
\hline Trayamar & $20-25$ & 3,5 & Pendiente \\
\hline Trayamar & $20-25$ & 3,8 & Pendiente \\
\hline Trayamar & 20 & 3,9 & Pendiente \\
\hline Trayamar & 20 & 3,4 & Pendiente \\
\hline Trayamar & 8 & 1,9 & Pendiente \\
\hline Trayamar & $5-10$ & 2,0 & Anillo \\
\hline Trayamar & 20 & 3,5 & Cuenta \\
\hline Trayamar & $15-20$ & 3,7 & Cuenta \\
\hline
\end{tabular}


La diferente concepción de la orfebrería entre los pueblos mediterráneos y los del noroeste peninsular encuentra un sólido argumento en la propia composición del metal base, como se recoge en la figura 4. Mientras en los materiales del noroeste predomina el oro con alta carga de plata con valores superiores al $20 \% \mathrm{Ag}$, en el área mediterránea los valores se sitúan en el extremo contrario del gráfico dada su cualidad de poco o nada argentados. En un principio nos planteamos qué relación podría existir entre los tipos de objeto y la aleación para de ese modo ofrecer una explicación al fenómeno. Como es sabido, la orfebrería del noroeste se caracteriza por sus objetos pesados (torques, brazaletes, grandes fíbulas), en tanto que las joyas ibéricas son piezas livianas, de unos pocos granos de peso (pendientes, aretes, anillos). Cabía la posibilidad de pensar en un ahorro sustancioso de oro en los objetos pesados a base de incrementar la ley de plata, reservando para los pequeños el oro de mejor calidad. Sin embargo no parece que esta pueda ser la explicación correcta pues entre los análisis del noroeste hay algunas joyas pequeñas, como un pendiente de La Graña y una cuenta de collar de Elviña, con más del $20 \%$ de plata, dejando muy en entredicho la suposición anterior. Tampoco podemos apoyar las pesadas piezas de oro argentado del noroeste en ninguna tradición previa pues por el momento no se ha apreciado en ningún período procedente una tendencia de tal naturaleza:

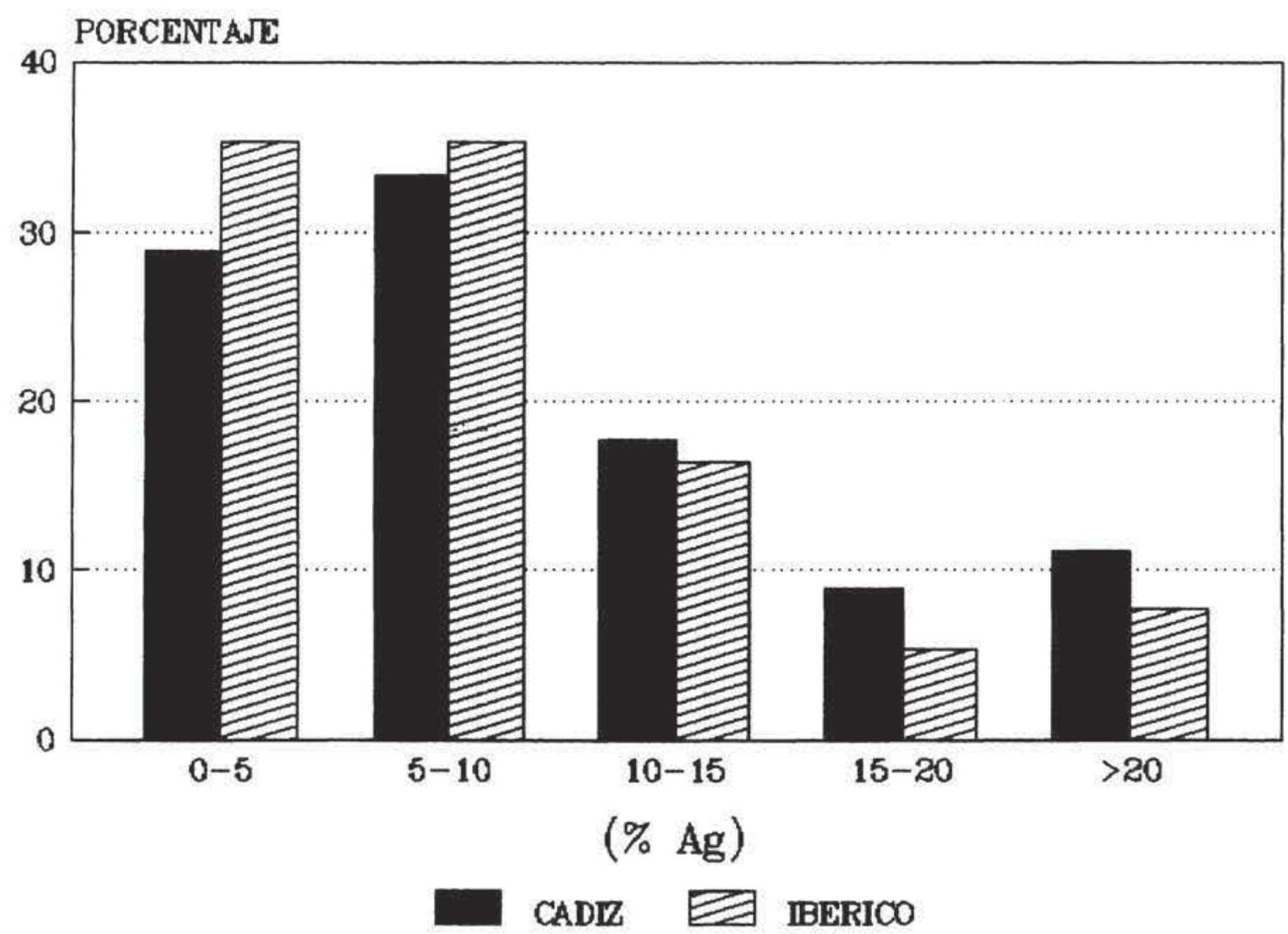

Figura 3. Contenido en plata (Frecuencias relativas). 
el mismo tipo de objetos, fueran o no pesados, se elabora con oro de composiciones muy diversas o, lo que es lo mismo, no se detecta ninguna relación entre el peso y el tipo de aleación. Parece razonable concluir, por tanto, que la diferencia entre ambas regiones responde a una tradición cultural y tecnológica distinta.

\section{AFINADO DEL ORO}

Si hacemos un repaso general a la evolución del contenido de plata como muestra la figura 5, llama la atención que después de reflejada la tendencia al aumento desde el Bronce Antiguo y Medio hasta el Bronce Final, tendencia que se agudiza durante el Hierro II en el noroeste, en el mundo mediterráneo e ibérico experimente un retroceso y a veces disminución drástica la plata ligada al oro, incluso por debajo de los valores detectados en el Bronce Antiguo cuando gran parte de la plata es debida a su asociación natural con el oro y las aleaciones son menos frecuentes que en los períodos posteriores. Este hecho llamativo debe

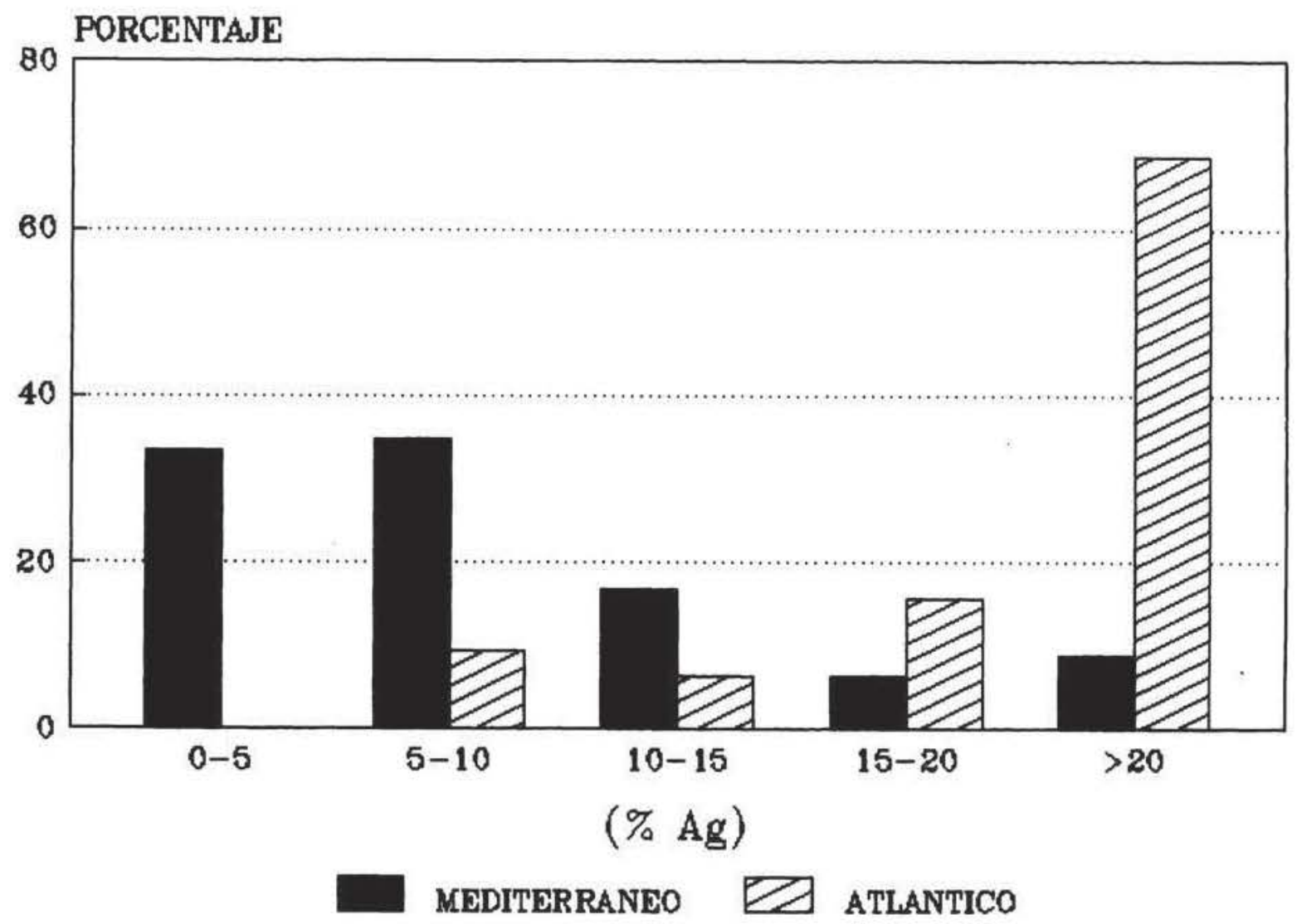

Figura 4. Contenido en plata (Frecuencias relativas). 
ligarse a la puesta en escena en las comunidades mediterráneas de una técnica para afinar el oro bruto, mediante la cual era posible eliminar la mayor parte de los metales a él asociados.

Los testimonios arqueológicos y numismáticos indican que la cementación del oro con sal común era conocida en Sardes (Anatolia occidental) desde tiempos de Creso (575-550 a. C.) ${ }^{1 x}$. Desde allí se difundió el mundo griego, empleándose para afinar oro en tiempos de Filipo II de Macedonia y de Alejandro Magno ${ }^{19}$. Posteriormente parece que se generaliza por todo el ámbito mediterráneo, quedando recogida en textos como el de Diodoro Sículo, donde describe el método usado por los egipcios del siglo II a. C. según la referencia de Agatharcides, y que consistía en envolver el oro con sal y arcilla, calentándolo en un horno durante cin-
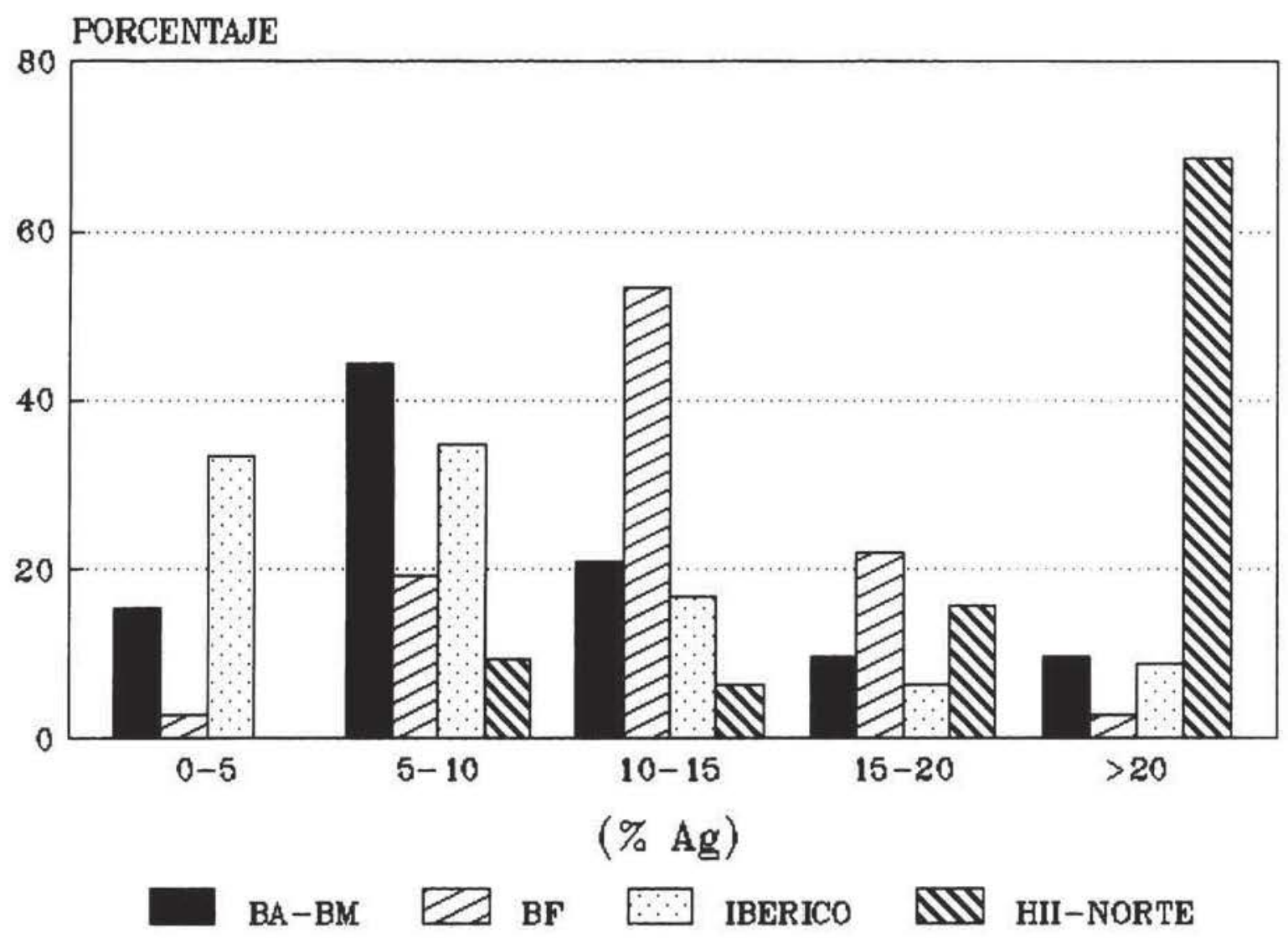

Figura 5. Contenido en plata (Frecuencias relativas).

18 J. P. GEALY, «Greek and Roman gold sources: the literary and scientific evidence», en Minería y metalurgia en las antiguas civilizaciones mediterráneas y europeas, vol. II, Ministerio de Cultura, Madrid, 1990, p. 13.

${ }^{19}$ HARTMANN y KalB, op. cit., p. 11, p. 98. 
co días ${ }^{20}$. Este debió ser el método practicado en el Imperio Romano ya que Plinio el Viejo describe un sistema similar para el afino del oro en Hispania, aunque con la variante de añadir pirita de cobre ${ }^{21}$.

Por la cronología de las piezas peninsulares que denuncian claramente el uso de oro afinado la técnica pudo conocerse en las costas alicantinas desde, al menos, el siglo IV a. C., y deberse a influencias griegas. El caso más claro es el tesoro de Jávea, fechado según Almagro Gorbea en dicho siglo IV ${ }^{22}$, y que estilísticamente responde a un influjo helénico, habiéndose planteado incluso la posibilidad de que pudiera ser obra de un artesano griego. El empleo en su manufactura de oro afinado corrobora esa posible relación o influencia. La distribución de joyas de oro afinado coincide con los territorios ibéricos, resultando un argumento a sumar a los otros muchos que sustentan el influjo griego sobre la cultura ibérica. Hay, sin embargo, ciertos elementos discrepantes que distorsionan este panorama feliz: el tesoro de La Aliseda tiene un anillo de oro sin plata y con un $6 \%$ de cobre que podría ser de oro afinado ligado con cobre, y otras piezas con poca plata que podrían serlo también. Si así fuera, y dado que este tesoro se fecha a fines del siglo vil a. C., cabría cuestionar la cronología anatolia antes apuntada, remontándola cómodamente hasta el punto de hacerla compatible con la arribada de elementos fenicios (como sucede, por ejemplo, con el hierro), o cambiar el centro de invención del afinado del oro. Mucho más prudente resulta por el momento considerar el anillo de La Aliseda una aleación excepcional (fortuita?) oro-cobre, porque el resto de piezas poco argentadas, si bien no son frecuentes, tampoco son rarezas. Queda, finalmente, otra opción cual es que el tesoro de La Aliseda no constituye un conjunto unitario fechable todo él en el siglo vil, sino que algunas piezas sean de cronología posterior a la antes apuntada, dadas las circunstancias del hallazgo. Serán los nuevos análisis de orfebrería orientalizante los que posibiliten otros planteamientos.

En cambio los orfebres del noroeste no reflejan ningún indicio del empleo de la técnica de afinado, lo cual explicaría las diferencias compositivas constatadas y podría considerarse como un rasgo cultural diferenciador a la par que indicativo de la falta de contactos y relaciones fluidas con el sur de la Península.

A través de las composiciones podemos observar algún contraste más, probablemente relacionado con el proceso de afinado del oro. En la zona levantina, y más concretamente en las provincias de Alicante y Murcia, más del $60 \%$ de las joyas analizadas ofrecen una tasa de cobre mayor o igual que la de plata, pero nunca superando la cifra del $10 \% \mathrm{Cu}$. Materiales con estas características se dan esporádicamente en las provincias limítrofes y en Cádiz (figura 6). El oro fino, por ser de alta pureza, podía alearse con plata y cobre a voluntad controlando de ese modo el orfebre la ley del producto resultante - como sucede en la actualidad-, además de mejorar las propiedades del metal para trabajarlo mejor, como se ha dicho más atrás. En la descripción que hace el monje alquimista Theophilus del método de

\footnotetext{
${ }^{20}$ TYLECOTE, oip. cit., n. 5 , p. 87.

${ }^{21}$ HEALY, op. cit., n. 19, p. 14. La pirita y la sal común permitirían la formación de ácido sulfúrico y clorhídrico, quienes atacarían la plata y otras impurezas del oro depurando el metal noble.

${ }^{22}$ M. ALMAGRO GORBEA, «Orfebrería orientalizante», en El oro en la España prerromana, Revista de Arqueología, 1989, p. 68-81.
} 
afino por cementación con $\mathrm{sal}^{23}$ se insiste en la conveniencia de, una vez logrado el oro fino, alearlo con una pequeña cantidad de cobre. Theophilus no explica la razón, que probablemente no es otra que rebajar significativamente el punto de fusión de la aleación resultante. Las cantidades de cobre detectadas en las piezas ibéricas, en el tesoro de Jávea e, incluso, en algunas de La Aliseda, proporcionalmente mayores que las de plata en cada caso, sólo pueden deberse a la adición por separado de dicho metal e indica que los antiguos orfebres conocían el efecto. En otros materiales mediterráneos en los que la tasa de plata es siempre

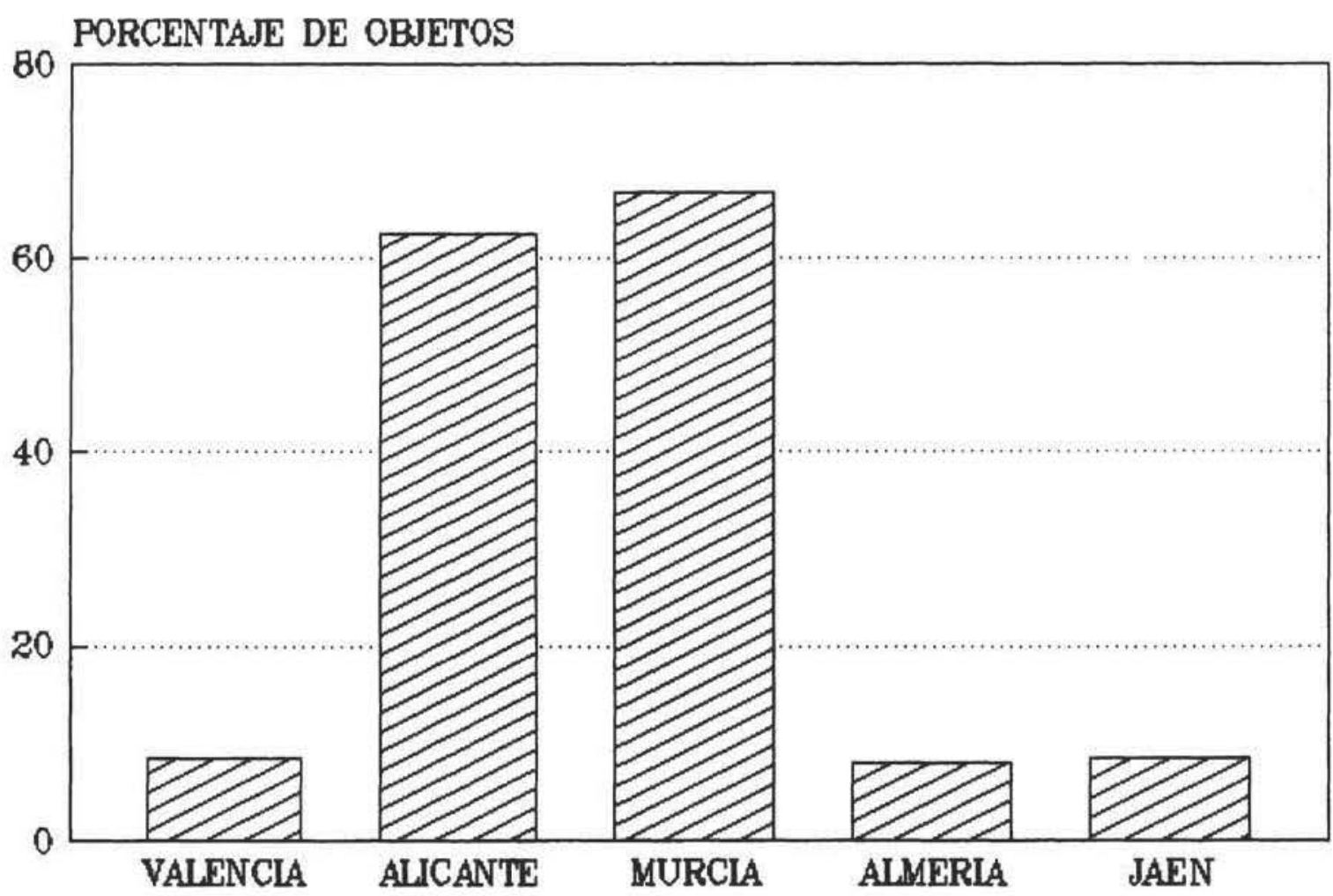

Figura 6. Objetos de oro ibéricos con $\% \mathrm{Cu}>=\mathrm{Ag}$

${ }^{23}$ THEOPILUS, On divers arts, Dover Publications, New York, 1979, pp. 108-109. Aunque mucho más tardía (comienzos del siglo XII), la técnica descrita por Theopilus no difiere sustancialmente de la empleada en la Antigüedad, con la ventaja en este caso de ser expuesta por un verdadero experto. Los textos clásicos (Agatharcides, Diodoro Sículo, Estrabón, Herodoto, Plinio, Papiro de Leyden), no fueron redactados por especialistas en el tema en cuestión (a excepción, quizá, de Plinio) y ello dificulta en cierto modo su comprensión y hace necesario expurgarlos de elementos incongruentes que acompañan al cuerpo fundamental (oro laminado o en porciones pequeñas, arcilla, sal, natrón, horno, tiempo de cementación). Así, la reproducción experimental del método propuesto por Diodoro Sículo resultó un fracaso (citado por HEALY, op. cit. n. 19, p. 13). Ningún texto antiguo hace referencia a la adición de cobre al oro fino, sin embargo era práctica común como demuestra un buen grupo de análisis de orfebrería. El metal para amonedaciones es otra cuestión, si de lo que se trata es de emplear oro puro (el aurei romano, con más del $99 \%$ de pureza). 
superior a la de cobre, este último podría ingresar en la liga por medio de una aleación platacobre de proporción 2:1 ó 4:1. Sea cual fuere la explicación, por el momento es constatable una concentración singular de materiales en Alicante y Murcia, respondiendo quizá a un rasgo tecnológico local o a una tradición artesanal más fielmente seguidora de ciertos cánones de afino del oro. 